\title{
Normal Tension Glaucoma: A Different Disease?
}

\author{
Kuldev Singh \\ Professor of Ophthalmology, Director, Glaucoma Service, Stanford University School of Medicine, USA
}

\begin{abstract}
The diagnosis and treatment of normal tension glaucoma remain controversial. The diagnostic dilemma is further compounded by the vast array of investigative modalities available. Also the concept of target IOP and physical quality of life of the patient is an important determinant of current glaucoma practice. This review provides a logical algorithm for the diagnosis, investigation and management of normal tension glaucoma (NTG).
\end{abstract}

\section{INTRODUCTION}

Von Graefe’s proposal that glaucomatous optic nerve damage could occur in eyes without high intraocular pressure (IOP) approximately 150 years ago led to such severe criticism that he ended up recanting his views. ${ }^{1}$ Schnabel, a half century later, supported von Graefe's initial position that optic nerve susceptibility to intraocular pressure varied significantly between patients. ${ }^{2}$ There were several reasons which led to Schnabel's views on this topic being accepted at the time that they were, the most important of which was the introduction of tonometry. While tools to accurately measure intraocular pressure confirmed the views of von Graefe and Schnabel in the 20th century, many practitioners continued to consider patients with "normal” IOP and glaucomatous optic nerve damage as having high intraocular pressures (IOPs) outside of office hours or burnt out "high pressure" glaucoma, concepts that persist to this date. Several glaucoma prevalence surveys from throughout the world subsequently revealed that glaucomatous optic nerve damage at "normal” IOPs was more common than once believed. ${ }^{3-7}$ For example, a large population based survey of glaucoma in East Baltimore, Maryland during the 1980's showed that approximately 50\% of all patients found to have "primary open angle glaucoma” had initial IOP measurements of less than $21 \mathrm{~mm} \mathrm{Hg}$, and further, approximately $20 \%$ had IOPs less than $21 \mathrm{~mm} \mathrm{Hg}$ on the first three measurements. $^{4,5}$

The aim of the landmark Glaucoma Research Foundation supported Collaborative Normal Tension Glaucoma Study (CNTGS) in the 1980's and 90's was to answer the following question: "Is IOP-lowering therapy appropriate in patients with normal tension glaucoma?" 8,9

Prior to this study, most ophthalmologists attempted to lower IOP in patients diagnosed with normal tension glaucoma but there was no proof that this was beneficial and there was a significant minority in the ophthalmic community who believed in avoiding further IOP lowering in such patients. In CNTGS, one eye of 145 subjects with IOPs in the "normal" range with no more than one reading as high as 23 or $24 \mathrm{~mm} \mathrm{Hg}$ and a median IOP of under $20 \mathrm{~mm} \mathrm{Hg}$ was randomized to treatment or observation and all eyes were treated if either progression was noted or fixation threatened. The treated group achieved a 30\% reduction in IOP using medications, laser and surgery. Over half of the patients in the study were able to achieve such IOP lowering without the need for laser trabeculoplasty or surgery. No beta-blockers or other adrenergic agents were used, largely because of concern that these drugs may negatively impact ocular or optic nerve blood flow resulting in further glaucomatous damage. While this hypothesis remains unproven, there has been a recent resurgence of interest in the concept of low ocular perfusion pressure being a risk factor for glaucoma progression. ${ }^{10-12}$ The treated group had a mean $12 \%$ risk of glaucomatous progression, whereas the untreated group had a 35\% risk of progression over the approximate 8 years follow-up period. While IOP lowering therapy reduced the risk of further visual field progression in patients with normal tension glaucoma, the trade-off included a greater risk of needing cataract surgery in the treated versus the control group. It is noteworthy that the benefits of visual preservation in the IOP lowering group were found to be statistically significant only when the effect of cataract was corrected for. While the study elucidated risk factors for progression such as disk hemorrhage, migraine headache, female gender and non-Asian ethnicity, it did not explain why a substantial number of patients with normal tension glaucoma did not progress, even without treatment.

The CNTGS was the first large multicenter randomized clinical trial to prove that IOP lowering therapy was effective in preventing the progression of open angle glaucoma. Ironically, this breakthrough occurred in patients with "normal” pressures and confirmed the view of many but not all that there should be no distinction made between the diagnoses of primary open angle and normal tension glaucoma. In the years that followed, other large multicenter randomized clinical trials confirmed the benefit of IOP lowering therapy in patients with elevated or higher than average IOP. ${ }^{13-16}$

How one makes the diagnosis and treats normal tension glaucoma, remain controversial. The approach favored by the author is one where the emphasis on determining how aggressively one should lower IOP in patients with open angle 
glaucoma depends more upon the historical rate of disease progression than the level of baseline IOP. As approximately half of untreated eyes in the CNTGS showed no progression over almost a decade, many patients categorized as having "normal tension glaucoma" would perhaps have been better classified as "glaucoma suspects" until they demonstrated that their condition had a natural history commonly seen with open angle glaucoma. This point, could, of course, also be made for patients with open angle glaucoma with "high" IOPs but all other things being equal, it is intuitively obvious that one would be more likely to offer initial IOP lowering therapy to a patient with apparent optic nerve damage, without prior demonstration of glaucomatous progression, when the IOP is high relative to when it is low. In patients with glaucomatous optic nerve damage and visual field loss who are newly diagnosed with such findings without prior information allowing determination of rates of progression, a "high" IOP should generally be lowered. In patients with the same degree of damage and historical as well as current information showing "normal" or average IOP, particularly when it is in the low normal range, it may commonly be best to hold off on initiating IOP lowering therapy while assessing the natural history of the condition over time. Many such patients will show minimal if any measurable progression over time suggesting that they have a condition with a natural history which is different than that commonly seen with open angle glaucoma. ${ }^{17}$ Complicating matters further is the fact that different populations have varying "normal" IOPs with the Japanese, for example, having lower IOPs, on average, than Caucasians. Intraocular pressure should, of course, be judged as being normal or abnormal in the context of the mean and variability in the particular population being examined. ${ }^{6}$ Unfortunately, however, such population information is currently not available in many regions of the world.

The situation is completely different in patients who have already demonstrated disease progression consistent with the natural history of glaucoma. Some might say that all statistical progression on automated visual field testing, for example, indicates that glaucoma is present and is getting worse. An alternative view might be that in areas where there is already damage, aging changes which result in ganglion cell death and retinal nerve fiber degeneration, may lead to statistical progression that does not necessarily indicate a glaucomatous process. ${ }^{18}$ Nevertheless, progressive glaucomatous optic nerve damage, with or without corresponding visual field loss, is generally treated with further IOP lowering therapy, regardless of the level of IOP. In such patients who have demonstrated progression, the author's treatment approach is no different for normal tension glaucoma patients relative to those with primary open angle glaucoma. While an arbitrary IOP reduction from baseline such as $30 \%$ is recommended by some, the target IOP concept minimizes the importance of side effects, and the risks of therapy often become progressively greater as we try to achieve very low IOPs. ${ }^{19}$ It is difficult to get IOPs in the single digits with medical therapy, for example, and surgical therapy is associated with substantial risks including those related to hypotony. The degree of desired IOP lowering should be based upon the expected risks and benefits of each treatment step, taking into consideration the severity of disease, the documented rates of progression, patient age, which may be an indicator of life expectancy, and the patient's willingness to accept the risks associated with therapy. These principles should apply regardless of whether or not the IOP is average, above average or below average.

The approach that will ultimately replace the target IOP concept is one where therapy is advanced until the expected marginal benefit (IOP lowering) of the next step is outweighed by the expected risks of the next step, taking the patient specific factors mentioned earlier into consideration. ${ }^{20}$ This is a dynamic process as the expected benefits and risks of a therapeutic option for a particular patient can change over time. For example, the additional benefit of achieving a single digit IOP in a patient with mild glaucomatous optic nerve disease and an IOP in the mid teens may be small. If over time, however, that same patient develops further glaucomatous damage and now has split fixation with IOPs in the mid-teens, the marginal benefit of lowering IOP to single digits may be greater than it had been when the disease was less severe. Glaucoma filtration surgery may be entertained at this stage when it would not have been considered at the previous less advanced stage. Most saavy practitioners who currently claim to use the target IOP concept will generally change the target when they are faced with such dynamic scenarios. Unknowingly, they are actually ignoring the target IOP concept and basing treatment on marginal benefits and risks of additional steps. These principles, of course, apply for patients at any baseline IOP level and there is no distinction between primary open angle and normal tension glaucoma.

While some have advocated a complex diagnostic work up in patients with normal tension glaucoma, such an approach is generally not necessary if one is confident that the optic nerve damage appears glaucomatous. Other potentially lifethreatening entities such as central nervous system (CNS) tumors can result in conditions masquerading as glaucoma, but these entities are rarely linked to IOP and thus the decision to proceed with further diagnostic testing in glaucoma suspects in an effort to find such entities, should not, in my opinion, be based primarily upon the level of IOP but rather on other factors that lead the physician to suspect a non-glaucomatous process. The IOP-glaucomatous optic neuropathy relationship is highly variable between subjects in any and all populations and thus the level of IOP is neither sensitive nor specific in making distinctions with regard to which patients should have CNS imaging to rule out non glaucomatous conditions. ${ }^{18}$ Careful examination of the optic nerve, neurologic examination and other tests are better suited to make such distinctions. 
We have come a long way since the myth of $21 \mathrm{~mm}$ Hg when glaucoma was considered primarily a disease of IOP with a single level defining the disease and determining the success and failure of therapy. But somehow, many still cling to the belief that there is something fundamentally different about patients with glaucomatous optic nerve disease who have so called "normal" intraocular pressures relative to those with higher than average IOP. This myth may take another century to refute, but there is no doubt that the misconception will ultimately be set right.

\section{REFERENCES}

1. von Graefe A. Amaurose Mit Sehnervenexcavation. Graefe’s Arch Clin Exp Ophthalmol 1857;3:484.

2. Schnabel WJ. Klinische Daten zur Entwicklungsgeschichte der Glaucomatosen. Zeitschr Augenheilkd 1908;19:335.

3. Banks JLK, Perkins ES, Tsolakis S, Wright JE. Bedford Glaucoma Survey. Br Med J 1968;30:791-79.

4. Sommer A, Tielsch JM, Katz J, et al. Relationship Between Intraocular Pressure and Primary Open Angle Glaucoma Among White and Black Americans. Arch Ophthalmol 1991;109: 1090-95.

5. Tielsch JM, Katz J, Singh K, et al. A Population Based Evaluation of Glaucoma Screening: The Baltimore Eye Survey. Am J Epidemiol 1991;134:1102-10.

6. Shiose Y, Kitazawa Y, Tsukahara S. The Epidemiology of Glaucoma in Japan: A Nationwide Glaucoma Survey. Jpn J Ophthalmol 1991;35:133-55.

7. Quigley HA, West SK, Rodriguez J, et al. The Prevalence of Glaucoma in a Population-Based Study of Hispanic Subjects, Proyecto Ver. Arch Ophthalmol 2001;119:1819-26.

8. The Collaborative Normal Tension Study Group. Comparison of Glaucoma Progression Between Untreated Patients With Normal Tension Glaucoma and Patients With Therapeutically Reduced Intraocular Pressure. Am J Ophthalmol 1998;126: 487-97.

9. The Collaborative Normal Tension Study Group. The Effectiveness of Intraocular Pressure Reduction in the Treatment of Normal Tension Glaucoma. Am J Ophthalmol 1998;126: 498-505.

10. Tielsch JM, Katz J, Sommer A, et al. Arch Ophthalmol 1995;113:216-21.
11. Leske MC, Wu SY, Nemesure, et al. Incident Open-Angle Glaucoma and Blood Pressure. Arch Ophthalmol 2002;120:95459.

12. Leske MC, Heijl A, Hyman L, et al. Predictors of Long-term Progression in the Early Manifest Glaucoma Trial. Ophthalmol 2007;114:1965-72.

13. AGIS Investigators. The Relationship Between Control of Intraocular Pressure and Visual Field Deterioration. Am J Ophthalmol 2000;130:429-40.

14. Kass MA, Heuer DK, Higginbotham EJ, et al. The Ocular Hypertension Treament Study: A Randomized Trial Determines That Topical Ocular Hypertensive Medication Delays or Prevents the Onset of Primary Open-Angle Glaucoma. Arch Ophthalmol 2002;120:701-13.

15. Leske MC, Heijl A, Hussein M, et al. For the Early Manifest Glaucoma Trial Group. Arch Ophthalmol 2003;121:48-56.

16. Lichter PR, Musch DC, Gillespie BW, et al. Interim Clinical Outcomes in the Collaborative Initial Glaucoma Treatment Study Comparing Initial Treatment Randomized to Medications or Surgery. Ophthalmol 2001;108:1943-53.

17. Doshi A, Kreidl KO, Lombardi L, Sakamoto, D and Singh K. Nonprogressive Glaucomatous Cupping and Visual Field Abnormalities in Young Chinese Males. Ophthalmol 2007;114:472-79.

18. Frisen L. High Pass Resolution and Age Related Loss of Visual Pathway Neurons. Acta Ophthalmol 1991;69:511-15.

19. Singh K, Spaeth G, Zimmerman T, Minckler D. Target IOP: Glaucoma’s Holy Grail. Ophthalmol 2000;107:629-30.

20. Singh K, Shrivastava A. Early Aggressive Intraocular Pressure Lowering, Target Intraocular Pressure and a Novel Concept for Glaucoma Care. In press, Survey of Ophthalmology.

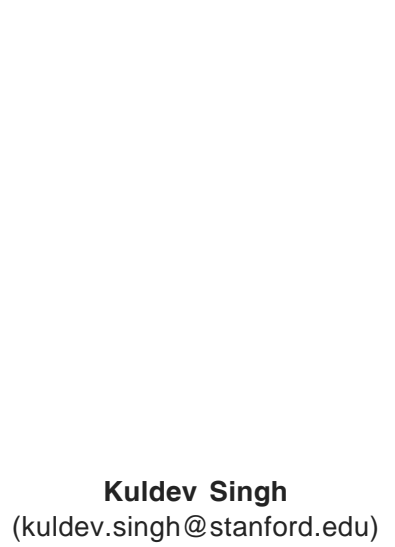

Fear of the pain blinds us to the goal of healing. Only by seeing our problems clearly and experiencing them can we do something about them. -Bob Hoffman 